\title{
“MODIFIED" THORACOPLASTY
}

\author{
BY \\ T. HOLMES SElloRS, J. W. JACKSON, AND J. G. CALlANAN \\ From the London Chest and Harefield Hospitals
}

(RECEIVED FOR PUBLICATION APRIL 29, 1955)

Thoracoplasty has been for many years accepted as the standard collapse operation in the treatment of pulmonary tuberculosis, and as an operation it has undergone surprisingly little change or variation. There have, however, been periodic modifications in technique designed to overcome some of the disadvantages or to enhance some of the advantages of the procedure, but these have proved of little lasting value unless they have followed the principle of concentric relaxation upon which any major collapse operation is based.

The original thoracoplasty was the Sauerbruch paravertebral operation, which was evolved after a period of trial and error. This was a "total" operation producing collapse of the whole of one lung. It was originally practised without pneumolysis, which was introduced by the Scandinavian school, who also tended to reduce the extent of the collapse and to make it a "partial" or " selective" collapse over the apex. In the 1930s a number of modifications were introduced. Gravesen, using an extrapleural pneumolysis and a limited rib resection, designated his form of operation as a "cupola-plasty," and Romanis and Sellors (1936) reported a limited type of operation referred to as a "strip-plasty," but, of all the procedures evolved, the extra-fascial pneumolysis and partial thoracoplasty that bears Semb's name (1935) is the one that has been widely adopted and accepted. Not even the current enthusiasm for pulmonary resection has obscured the popularity of this operation.

There are, however, certain disadvantages which can briefly be stated as follows. First, in any thoracoplasty which requires the removal of more than three or four ribs the procedure has to be performed in two or more stages, with resulting physical and psychological distress to the patient. Secondly, there is the problem of deformity in the form of a scoliosis resulting from the disturbance of muscle balance on each side of the vertebral column. This should not be of any consequence if appropriate physiotherapeutic measures are available, but it is undoubtedly worse if the first rib is removed when the scalene and other attached muscles are divided. The next point is that once the apex of the lung has been freed from the parietes its movement is paradoxical. A limited degree of paradoxical movement is not significant, but if there is any atelectasis or sudden coughing a benign state of affairs rapidly becomes "malign" with serious disturbance.

Finally, there is the problem of re-expansion of the collapsed lung. This occurs in a proportion of cases some time after the operation, the collapsed or depressed apex "creeping" upwards towards its original position. Middle-aged patients with a persistent cough and emphysema are often victims of this circumstance. Control of this creeping tendency, which is most noticeable at the mediastinum, is usually countered by suture of intercostal muscle bundles over the apex, postoperative air filling of the dead (Semb) space, bone chips, foreign bodies, and so on.

If an operation can be devised which is a onestage procedure, which can be performed without removing the first rib, and which maintains the lung apex permanently at the level of the hilum, the advantages are obvious. The procedures recommended by Holst (1952), Björk (1954), and Brock (1955) fulfil these criteria, and the operation which is being introduced in this article comes under the same category.

This operation, which we here refer to as a "modified" thoracoplasty, has been used for a number of years in well over a hundred patients and has proved to be entirely satisfactory for the purposes for which it was intended. It consists of forming a rib flap, which is turned inwards and secured to the mediastinum after an extensive pneumolysis. The first rib is left in place and the second, third, and fourth ribs are divided front and back without being removed, and the newly attached flap forms a firm roof which effectively prevents re-expansion or "creeping" of the lung. The procedure is only designed for cases in which the disease is comparatively limited, but it can also 
be used as a first stage to a more extensive operation.

In cases of pulmonary excision the formation of a firm apex at a lower level is of considerable value where some collapse is required in addition to the resection.

\section{TECHNIQUe}

The incision and exposure are the same as for a standard thoracoplasty. A J-shaped skin incision with division of the latissimus dorsi and trapezius and rhomboid muscles is followed by retraction of the scapula. The posterior ends of the second, third, fourth, and sometimes fifth ribs are exposed, and 4 to $5 \mathrm{~cm}$. is resected subperiosteally as close to the transverse processes as possible. The corresponding intercostal bundles, including the arteries and nerves, are divided between ligatures and then the lateral border of the first rib is exposed. The periosteum is stripped off the under-surface of the first rib as far forward as the costal cartilage; a little additional dissection exposes the apex of the lung. Apicolysis is then carried out from above and behind on to the mediastinum until the second costal cartilage is visualized. This is divided, as also the third costal cartilage with the anterior intercostal tissues. The fourth rib is divided in the axillary line and the fifth a little posterior to this. A large flap of the upper chest wall is thus formed, hinged below on the fourth or fifth intercostal muscles. The posterior edge is the posterior end of the ribs and in front the second and third costal cartilages are free, though the fourth and fifth have still intact anterior intercostal muscles. The upper border of this flap is the first intercostal muscle with the attached second rib.
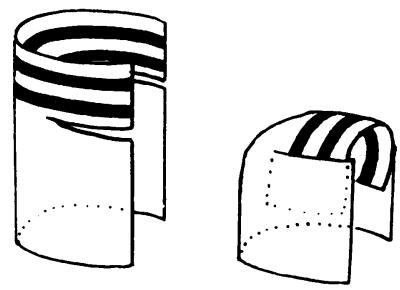

FIG. 1.-Diagram showing the principle of the modified thoracoplasty with formation of a new roof over the lowered apex.

The mediastinal pneumolysis is continued and the apex mobilized down to the level of the lung root, but there is no necessity to carry out any dissection under the rib flap. When the apicolysis is complete the flap is turned inwards and downwards so that the upper border (second rib and first bundle) lies at the lung root against the mediastinum, and the whole flap forms a new roof

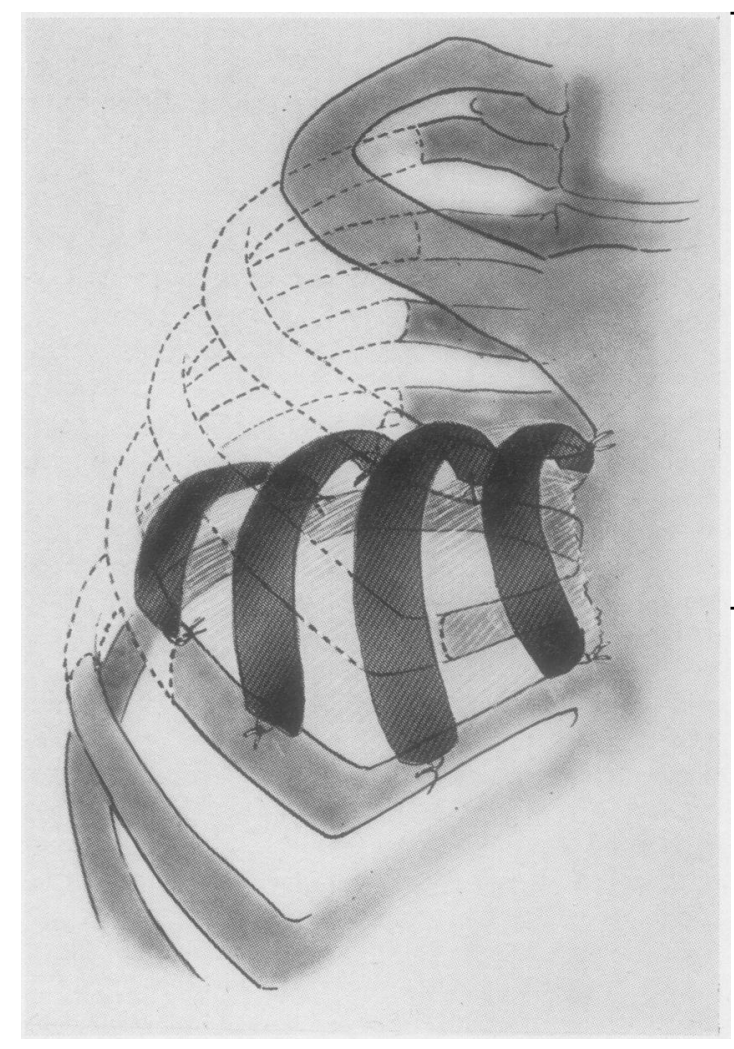

FIG. 2.-Dark sections show ribs which have been divided ard secured in new position.

over the depressed lung. Some " tailoring" of bone may be required to ensure that the flap fits against the mediastinum before fixing stitches are inserted.

The chief points of fixation are front and back, the former being more difficult to achieve. Posteriorly the periosteum on the lateral aspect of the sixth or seventh vertebra is transfixed by a stout, non-absorbable suture. This is passed $\frac{\text { ? }}{7}$ through the first and second intercostal muscles on $D$ the flap or through a hole drilled in the second rib. The third rib is fixed in the region of the $N$ transverse process and the fourth and fifth ribs attached more anteriorly. Anteriorly, the second costal cartilage is sutured to the mediastinum at $\underset{\omega}{N}$ the level of the third interspace. A transverse shelf of bone and muscle is now formed, but there 0 is still a gap between the mediastinum and the $\bar{\Phi}$ second rib. This is closed by five or six fine? sutures between the mediastinum and intercostal 0 muscle-a procedure which may require some ingenuity in avoiding nerves and vessels.

The end-result is a firm and complete horizontal roof, formed by the flap and held securely in 
position. The wound is closed in layers without drainage.

The above description refers to a collapse procedure alone, but if it is to be used in conjunction with lung resection it is somewhat modified. The incision is similar, and after division of the second, third and fourth ribs posteriorly, a small fourth intercostal incision is made. The division of the back ends of ribs and a very limited intercostal incision gives an adequate intrathoracic exposure. The front ends of the second and third ribs are divided from inside the chest, and the under-side of the first rib is stripped clear to set the flap free. After the resection is completed the flap is turned inwards and downwards on to the mediastinum and fixed as in the collapse operation. The mediastinal gap is more easily closed, since a mediastinal pleural flap can be mobilized for suture to the second rib and attached muscle. Intercostal vessels are only severed where they are necessary for exposure or for approximation of the flap to the mediastinum.

The operation can be carried out under local anaesthesia, but in nearly all cases a general anaesthetic has been used in conjunction with regional infiltration and a brachial plexus block. The degree of disturbance and shock to the patient is about the same as that in a first-stage thoracoplasty, but the problem of paradoxical movement of a "soft" or mobile apex does not arise as the flap constitutes an effective barrier. The operation is undoubtedly more complicated and difficult than a standard first-stage thoracoplasty, but the advantages of leaving the ribs untouched over the lateral aspect of the lung are considerable. Failure of the flap to hold in place is invariably due to technical errors in securing it anteriorly. In a number of cases the anterior pleural membrane is extremely thin and easily torn; if this occurs the defect should be closed and patched with muscle, but occasionally the tear is extensive and in this case a temporary water seal drain at the bottom of the free pleural cavity drains off the blood and effusion which gravitate from the operation area. An unrecognized pleural tear will lead to an effusion which requires early and repeated aspiration.

The vitality of the muscle and bone flap may well be questioned once the intercostal vessels have been divided, but in no instance has there been any suggestion of bone necrosis. Originally we attempted to leave the intercostal vessels intact, but this was not satisfactory if an efficient apicolysis was to be achieved, and there was often excessive tension on the vessels as the flap was turned downwards. It would appear that there is an adequate blood supply from the lower anterior intercostal vessels and from the vessels through the hinge of the flap to maintain nutrition of the second and third ribs whose normal blood supply is divided front and back.

\section{Complications and Results}

We have performed modified thoracoplasty on 135 patients. In 115 instances it was used as a collapse measure only, and in 20 patients it was used in conjunction with pulmonary resection. The comments and results are only concerned with the collapse operation.

In the 115 operations there were two deaths, one due to cerebral thrombosis and the other to an associated pulmonary stenosis whose severity was not recognized. There were three examples of reactionary haemorrhage into the dead space: these required aspiration and transfusion. The most common complication was a blood-stained pleural effusion caused by a leak of blood and serum through a hole or tear in the parietal pleura into the general pleural cavity. Sometimes this tear was recognized and more or less satisfactorily closed, but on occasions its presence was only inferred by dullness and loss of air entry at the base of the chest. Early and complete aspiration was effective in minimizing this complication, and, as has been already indicated, some cases were treated by closed drainage for the first two or three post-operative days.

The behaviour of the tuberculous process in the lungs was unexceptional. There was no instance of contralateral spread, and extension or reactivation of disease on the same side was rare. In only four cases was this latter complication suspected and only in two was it radiologically obvious. Atelectasis at the base on the same side as a thoracoplasty is a relatively frequent and important complication in the standard operation, but in the modified procedure it only occurred five times. It was treated by posture and bronchoscopy (where required) and resolved without incident.

Additional complications which developed some months after the operation were concerned with re-expansion of the lung apex. This occurred on four occasions and was almost certainly due to imperfect fixation of the flap to the mediastinum in the early stages of the development of the operation. In two of these cases a further operation was required to ensure adequate collapse. In three patients in whom the modified operation was used 
FIGs. $3 a$ and $3 b$.-Radiographs of patient with bilateral disease and cavitation at the right apex before and after modified thoracoplasty
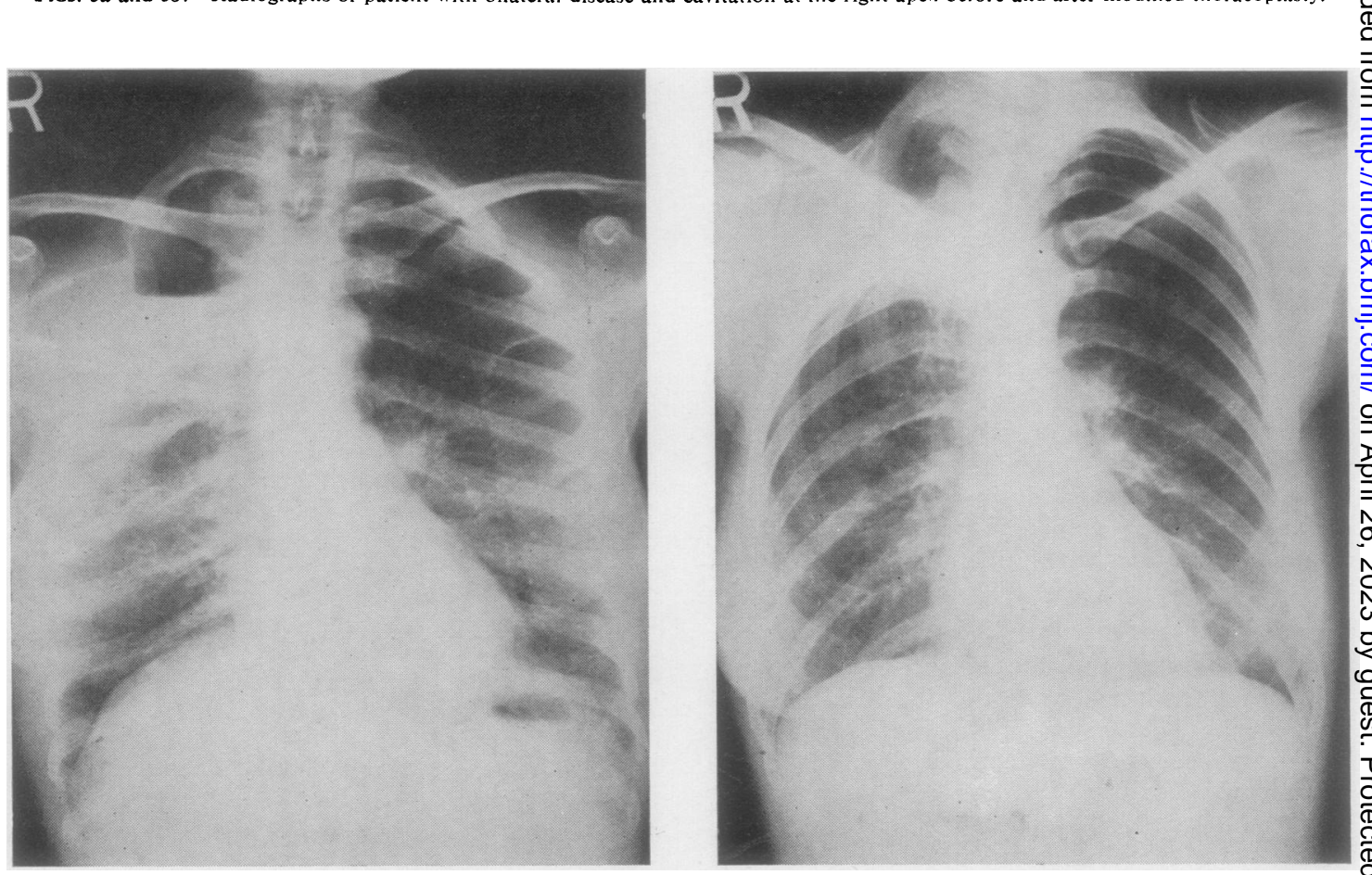

FIG. 4.-(a) Immediate post-operative radiograpn of modified tnviacuplasiy, anu ( $b$ ) appearance some weeks later.
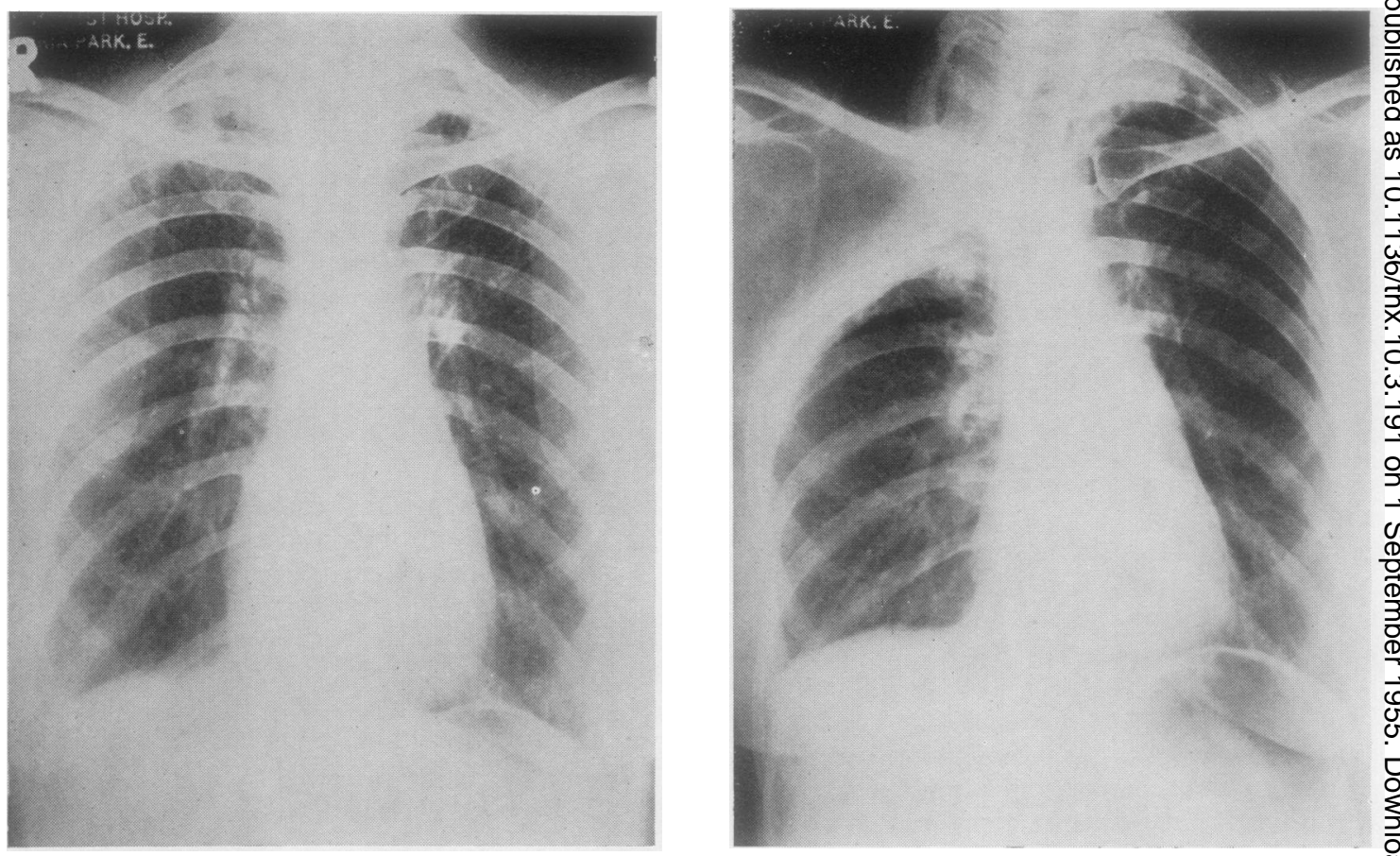


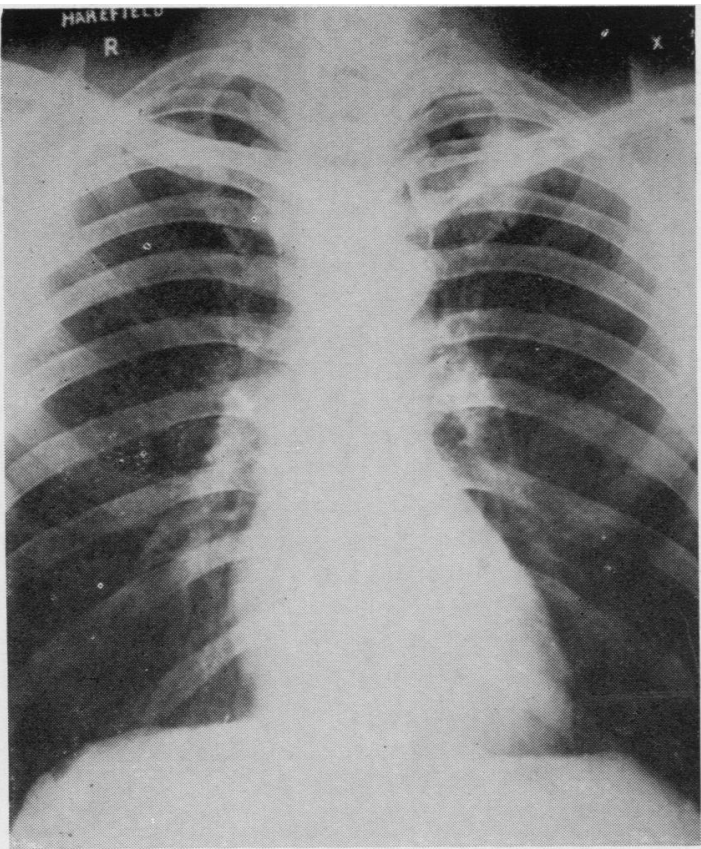

FIG. $5 a$.

Figs. $5 a, b$, and $c$.- Radiographs of a case of left apical disease in which the upper lobe was resected and a modified thoracoplasty carried out at the same time.

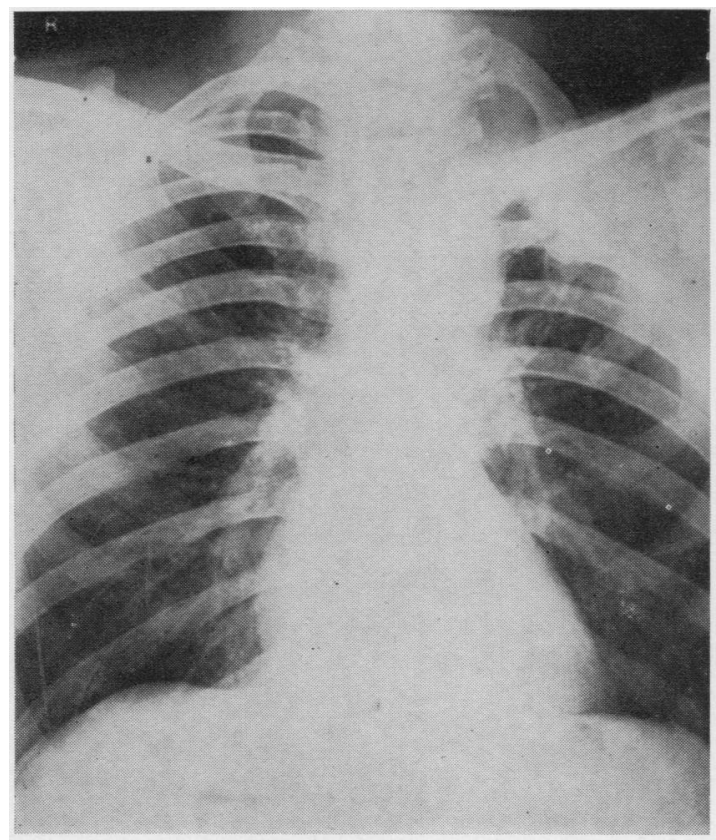

FIG. $5 c$. as a first stage, further rib resection was required. In two instances an upper lobectomy was performed nine months and one year after the operation for a breakdown of disease in the apex. It was a point of interest that in both cases there was no evidence of cavitation in the resected lobe and the breakdown had occurred in the anterior segment below the level of the collapse.

In pulmonary tuberculosis it is almost impossible to assess accurately the results of any form of treatment. There are so many variable factors that make for uncertainty. There is the individual reaction of the patient, the response to treatment, the behaviour of chemotherapy, and the whole host of imponderables that beset the course of the balance between infection and immunity. Next, there is the individual reaction of the surgeon

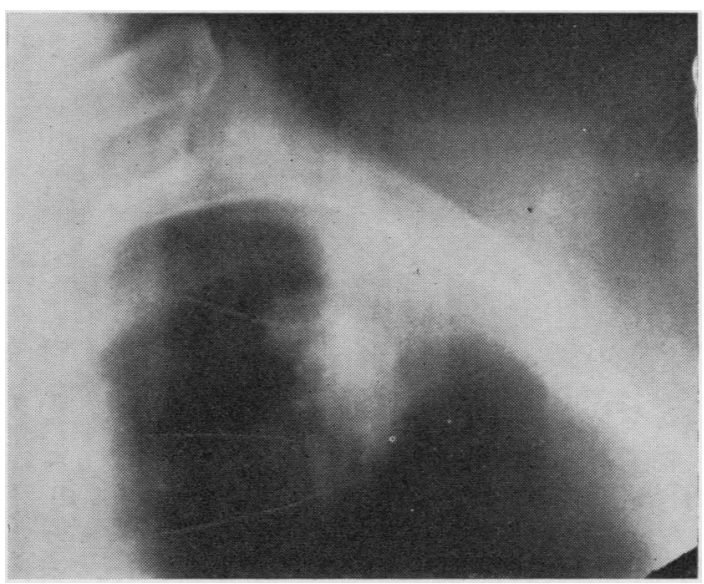

Fig. $5 \boldsymbol{b}$.

who may be actively or conservatively minded, or who may choose a different policy from one of his colleagues. All that can safely be asked of any given form of treatment is whether it produces the immediate object for which it was designed.

A follow-up has been carried out on the patients who have been operated on over a period varying between six months and four years, and in general the results suggest that there has been a considerable measure of success. Out of the 115 patients, 87 had had obvious cavitation, and in 62 of these there was an excavation of more than $2 \mathrm{~cm}$. diameter at the time of operation. Radiological closure was obtained in all instances. The sputum was positive for tubercle bacilli in 78 patients at the time of operation: this was converted in all cases except three, and in these there was obvious disease on the opposite side. 
Reference has already been made to exacerbation in the site of the operation. This occurred in two patients, who required resection at a later date, but these are the only instances in which instability of disease was obvious some time after collapse.

Re-expansion of collapsed lung has also been commented on, but in only two of these did this require further surgery.

In short, modified thoracoplasty has proved most satisfactory in the closure of small cavities and in conversion of sputum. It has rendered safe a latent smouldering focus, and has given a measure of insurance for the future.

\section{INDICATIONS}

The selection of patients for this form of operation has been left until last, since it is the most individual factor. In general the choice was confined to patients with limited and fairly stable disease, and there was a tendency to use it in young women (to minimize deformity) and in elderly emphysematous men whose lung might re-expand after collapse. Our classification gave six examples of advanced disease, 72 moderately advanced and 37 minimal. The disease was bilateral in 65 patients. An obvious cavity was present in $50 \%$ of the patients at the time of operation, and others had had cavities which had more or less responded to treatment. The presence of tubercle bacilli in the sputum is not an absolute indication for surgery, but in the presence of other features it has to be considered, and a positive reading was obtained in 78 cases of the operation series.

The figures give a rough idea of the type of cases selected-localized apical disease, well controlled, and with a cavity or a positive sputum. Extensive disease below the level of the sixth rib posteriorly is felt to be more suitably treated by a standard thoracoplasty assuming that collapse is to be preferred to excision. At the present time over the general run of cases requiring collapse nearly one half are treated by this modified procedure. On many occasions it is used as an insurance in a patient who has more than one breakdown in the apex, or who has bilateral disease? which can be controlled by hospital and drug regime, yet is not judged safe to lead a normaक active life. The value of a limited procedure is obvious in patients with bilateral disease, and aे combination of modified thoracoplasty on one side and artificial pneumothorax or excision on the other has proved a considerable advantage.

The question of age has already been touched on. A firm roof to the collapsed lung prevents the " creeping" tendency that is present in middle $\times$ aged or elderly men, and the absence of any $\vec{\rho}$ deformity is welcomed, particularly by young women with long necks. In a number of casese a more extensive collapse was planned, but the result obtained by the one-stage modified opera? tion was sufficiently good to avoid further surgery ${ }_{\mathcal{C}}$ In the world of tuberculosis where the patients are inconveniently knowledgeable about the treatment of their disease, this operation has become increasingly popular and on occasions an insis tence on its limitations has had to be put to the patients.

\section{SUMMARY}

A modification of the standard Semb thoraco plasty is introduced. It has been used 115 times

The basis of the operation is the formation of a bone flap consisting of the second, third, and fourth ribs. This is turned down and secured too the mediastinum after an extensive apicolysis has been performed. The technique, which is not dis similar to that of other operations based on the same principle, is described.

The risks and complications are slight and the early results are most gratifying. The indications are for limited forms of disease and where a permanent apical collapse is required.

The operation can also be adopted for use in conjunction with excision of lung.

\section{REFERENCES}

Björk, V. O. (1954). J. thorac. Surg., 28, 194.

Brock, R. (1955). Thorax, 10, 1.

Holst, J. (1952). Nord. Med., 48, 1288.

Romanis, W. H. C., and Sellors, T. H. (1936). Lancet, 1, 714. Semb, C. (1935). Acta chir. scand., 76, 561. 